Research Article

\title{
The Robustness Optimization of Parameter Estimation in Chaotic Control Systems
}

\author{
Zhen Xu* \\ Institute of Information Technology, Zhejiang Shuren University, Hangzhou, 310014, Zhejiang, China.
}

Received 20 September 2014; Revised 13 October 2014; Accepted 30 October 2014

\begin{abstract}
Standard particle swarm optimization algorithm has problems of bad adaption and weak robustness in the parameter estimation model of chaotic control systems. In light of this situation, this paper puts forward a new estimation model based on improved particle swarm optimization algorithm. It firstly constrains the search space of the population with Tent and Logistic double mapping to regulate the initialized population size, optimizes the fitness value by evolutionary state identification strategy so as to avoid its premature convergence, optimizes the inertia weight by the nonlinear decrease strategy to reach better global and local optimal solution, and then optimizes the iteration of particle swarm optimization algorithm with the hybridization concept from genetic algorithm. Finally, this paper applies it into the parameter estimation of chaotic systems control. Simulation results show that the proposed parameter estimation model shows higher accuracy, anti-noise ability and robustness compared with the model based on standard particle swarm optimization algorithm.
\end{abstract}

Keywords: Particle swarm optimization algorithm, chaotic control system, parameter estimation, double mapping constraint, evolutionary state identification, nonlinear decrease, genetic operator optimization.

\section{Introduction}

Chaotic motion is an objective and complex form of motion with ultimate boundedness and random regulation in nature [1]. At the beginning of the chaos phenomenon, the singularity, the high sensitivity to subtle change of initial conditions and instability make people feel it uncontrollable and try best to avoid it in production and experiment. People take it for grant that chaos does not possess the same synchronization as the periodic solution [2]. Recently, chaos science is interpenetrated with variety of disciplines else, such as biology, psychology, mathematics, physics, electronics, information science, astronomy, meteorology, economics, and even in music and art [3]. Among them, information security with synchronous chaos is most noticeable.

Chaotic control studies generally include chaotic control, synchronous control, and negative control [4]. Ditto et.al firstly used OGY method to stably control the fixed point on magnetic elastomers, but this method requires much time to test whether the system goes into the neighbourhood of control action without regularity [5]. On the basis of OGY method, Pyragas realized the control of unstable periodic obit of chaotic system with retardation of its state variables

* E-mail address: xuzheng355@163.com ISSN: 1791-2377 (c) 2015 Kavala Institute of Technology. All rights reserved. as the feedback [6]. The adaptive control method of chaotic system was proposed by Huberman firstly and applied bySinha into a system with multiple parameters and highdimensional nonlinearity. Under the condition of parameter mutation or dynamic alteration by disturbance, the adaptive control algorithm proposed by Sinha is efficient for recovering the system state [7]. Both of two methods are not suitable to control the motion state of the system to an unstable orbit, and their control rigidity is not easily determined and the initial value range of disturbance parameter is also largely limited. Vassiliadsa proposed a parameter adaptive control algorithm based on reference model and made the chaotic system index approaches to target state [8]. Besides them, there are Backstepping control, impulse control, sliding-model control, fuzzy control and neural network control, and some new ways like passive control and constrained control which have achieved great attention by many scholars [9]. The parameter estimation problem of chaotic system is an inverse problem of kinetic study with the data in test and operation and the system identification technology to build the mathematic model reflecting the essence of system and identify the undetermined parameters. This is the traditional grey box model [10]. Chen estimated the parameters of objective system with parameter adaptive method and achieved the generalized synchronization [11]. Yassne also reached the estimation and synchronization of unknown systematic parameters [12]. Wang raised a kind of parallel genetic 
algorithm for synchronous optimization of PID structure and parameter. Guan gave a concept of parameter identification observer, translating parameter identification problem into observation problem. Therefore, the design of observer is available for the parameter identification problem of the Lorenz system [13]. Gao et.al introduced improved PSO algorithm for the estimation and online estimation of Lorenz system [14]. Dai Dong constructed an appropriate fitness function which translated the parameter estimation problem to a parameter optimization problem with genetic algorithm [15].

In view of the problem that the parameter estimation model of current chaotic control system after adding noise has a low precision, this paper designed a parameter estimation model based on improved particle swam algorithm, with double mapping constraint strategy, evolution state judgement strategy, nonlinear decrease strategy, and crossover operator strategy to increase the robustness of this algorithm in chaotic control systems.

\section{The Shortcomings of PSO Algorithm in Chaotic Control Parameter Estimation}

The proposal of Lorenz chaotic system opened a new era for chaotic system theory and its application. Lorenz chaotic system is a dynamic system with singular attractor.

$\left\{\begin{array}{l}x^{\prime}=\sigma \cdot(y-x) \\ y^{\prime}=\gamma x-x z-y \\ z^{\prime}=x y-b z\end{array}\right.$

where parameters $\sigma=10, \gamma=28, b=8 / 3$ and this equation represents the chaotic system. A simple physical implementation is a circulation in heat convection tube where fluid is heated upside while cooled downside. At this time, $x$ is the fluid velocity, $y$ and $z$ is the perpendicular and vertical temperature difference; $\sigma$ is proportional to Prandtl number of fluid, $b$ is a constant about space, and $\gamma$ is proportional to Rayleigh number.

In terms of chaotic system, the known systematic parameter $\sigma$ and unknown parameter $b$ is usually studied. However, the dynamic information of unknown parameter is difficult to obtain. Therefore, it is supposed that $b^{\prime}=0$, and unknown parameter $b$ is seen as a state variable, then an augmented state variable $\left.\left[\begin{array}{lll}x & y & z\end{array}\right]^{T}, b^{T}\right]$ is acquired. Moreover, if all the state in equation (1) is available, only unknown parameters are required.

Particle Swarm Optimization algorithm (PSO), an evolutional computation method of swarm intelligence, is commonly used in numerical simulation. Suppose a target searching space of $D$ dimensional, the PSO randomly initializes a swarm with $m$ particles and the position $X_{i}$ of particle $i$ is represented as $\left\{x_{i 1}, x_{i 2}, \ldots, x_{i D}\right\}$. This position information is added into the optimization estimation function to get the fitness value for performance assessment of particles. Corresponding flight speed $V_{i}$ is denoted as $\left\{v_{i 1}, v_{i 2}, \ldots, v_{i D}\right\}$. In each iteration process, the particle updates its velocity and position by tracing two extreme values: one is the optimal value so far, namely individually extreme value $P_{i b e s t},\left\{P_{i b e s t .1}, P_{i b e s t .2}, \ldots, P_{i b e s t . D}\right\}$; the other is optimal solution of swarm particles, namely globally extreme value $P_{\text {gbest }},\left\{P_{\text {gbest. } 1}, P_{\text {gbest. } 2}, \ldots, P_{\text {gbest. } . D}\right\}[16]$.

Specifically speaking, in $k+1$ iterative calculation, particle $i$ updates its velocity and position with Eq. (2) and (3), with velocity limitation in Eq. (4) [17].

$$
\begin{aligned}
& v_{i d}^{k+1}=\omega \cdot\left[v_{i d}^{k}+c_{1} r_{1}\left(p_{i b e s t . d}^{k}-x_{i d}^{k}\right)+c_{2} r_{2}\left(p_{\text {gbest.d }}^{k}-x_{i d}^{k}\right)\right] \\
& x_{i d}^{k+1}=x_{i d}^{k}+v_{i d}^{k} \\
& \left\{\begin{array}{l}
\text { if } v_{i d}^{k+1}>v_{\max } v_{i d}^{k+1}=v_{\max } \\
\text { if } v_{i d}^{k+1}<v_{\min } v_{i d}^{k+1}=v_{\min }
\end{array}\right.
\end{aligned}
$$

where $i=1,2, \ldots, m ; d=1,2, \ldots, D ; \omega$ is inertia weight; $c_{1}$ and $c_{2}$ is accelerated factor; $r_{1}$ and $r_{2}$ is the random value uniformly distributed in interval $[0,1] ; v_{\max }$ and $v_{\min }$ is the extreme velocity.

Then, the estimation process of unknown parameter $b$ of chaotic system based on PSO algorithm is shown as follows.

1) Initialization.

$M$ individuals $P_{i}^{t}(k)(i=1,2, \ldots, M)$ of the initial population are randomly generated, $b_{\min } \leq P_{i}(t) \leq b_{\max }$ where $b_{\min }$ and $b_{\max }$ is the up and down limit, and $k$ is the iteration number.

2) Fitness value.

The corresponding state variable of individual $P_{i}^{t}(k)$ of generation $k$ is set as,

$\left(x\left(P_{i}^{t}(k)\right), y\left(P_{i}^{t}(k)\right), z\left(P_{i}^{t}(k)\right)\right)$

According to the measured systematic state variables $(x(t), y(t), z(t))$, the fitness value is calculated,

$f_{i}=\sum_{t=0}^{T}\left(\eta_{1}^{2}, \eta_{2}^{2}, \eta_{3}^{2}\right)$

where,

$\left\{\begin{array}{l}\eta_{1}=x\left(P_{i}^{t}(k)-x(t)\right) \\ \eta_{2}=y\left(P_{i}^{t}(k)-x(t)\right) \\ \eta_{3}=z\left(P_{i}^{t}(k)-x(t)\right)\end{array}\right.$

$t$ is a series of discrete-time sequence from 0 to $T$.

3) Optimization.

PSO algorithm is used for current population optimization.

4) Stop condition.

If $k \leq 100$, then step ends; otherwise, $k=k+1$, go back to step (2).

Generally speaking, in a pre-estimated unknown parameter interval, the solution space of objective function (6) is multimodal with complex structures. Comparing with other searching methods like hill climbing method, method of exhaustion and random search method, PSO algorithm has its unique advantages but still exists some defects. 
The estimation process of unknown parameter $b$ of chaotic system based on PSO algorithm is popularized and added by white noise, as follows,

$$
\left\{\begin{array}{l}
x^{\prime}=\theta_{1} \cdot(y-x) \\
y^{\prime}=-x z-\theta_{3} y \\
z^{\prime}=x y-\theta_{2} z
\end{array}\right.
$$

Constructing following system,

$$
\left\{\begin{array} { l } 
{ x ^ { \prime } = \theta _ { 1 } \cdot ( y - x ) } \\
{ y ^ { \prime } = - x z - \theta _ { 3 } y } \\
{ z ^ { \prime } = x y - \theta _ { 2 } z } \\
{ L = ( x , y , z ) }
\end{array} \rightarrow \left\{\begin{array}{l}
\tilde{x}^{\prime}=\tilde{\theta} \cdot(\tilde{y}-\tilde{x}) \\
\tilde{y}^{\prime}=-\tilde{x} \tilde{z}-\tilde{\theta}_{3} \tilde{y} \\
\tilde{z}^{\prime}=\tilde{x} \tilde{y}-\tilde{\theta_{2}} \tilde{z} \\
\tilde{L}=(\tilde{x}, \tilde{y}, \tilde{z})
\end{array}\right.\right.
$$

The objective function is taken as,

$$
f(\Theta)=\sum_{t=0}^{T}\|L(t)-\tilde{L}(t)\|_{2}^{2}
$$

PSO algorithm is used for parameter assessment $\Theta=\left(\theta_{1}, \theta_{2}, \theta_{3}\right)$. . We take $(10,10,10)$ as the initial point, and make it evolve to $T=100 h(h=0.01)$ to obtain the standard static variables $(x, y, z)$ at the discrete time sequence $0 h, h, \ldots, h, 100 h$ of the chaotic system of the unknown parameters. Therefore, standard PSO algorithm has bad robustness in the parameter estimation of the chaotic control system with noises. Thus, we make improvement on the PSO algorithm.

\section{The Optimization of PSO Algorithm}

\subsection{Population Initialization Based on Double Mapping}

In order to improve the accuracy of the parameter estimation, the population initialization should be firstly optimized to limit the search space of PSO algorithm. This paper takes the strategies of double mapping, Tent mapping and Logistic mapping, to improve it [18].

The sequence from Tent mapping is defined as,

$x_{n+1}=\left\{\begin{array}{l}\frac{x_{n}}{\sigma}, 0 \leq x_{n} \leq \sigma \\ \frac{1-x_{n}}{1-\sigma}, \sigma \leq x_{n} \leq 1\end{array}\right.$

where $\sigma \in(0,1)$, the sequence by Tent mapping has ergodicity and uniformity, and it is not sensitive to the initial value. When $\sigma=0.5$, the mapping subsection under this situation is shown below,

$$
x_{n}=\left\{\begin{array}{l}
4 x_{n}, 0 \leq x_{n} \leq \frac{1}{4} \\
2-4 x_{n}, \frac{1}{4} \leq x_{n} \leq \frac{1}{2} \\
4 x_{n}-2, \frac{1}{2} \leq x_{n} \leq \frac{3}{4} \\
4\left(1-x_{n}\right), \frac{3}{4} \leq x_{n} \leq 1
\end{array}\right.
$$

The definition of Logistic mapping is [19],

$a_{n+1}=f\left(a_{n}\right)=\mu \cdot a_{n} \cdot\left(1-a_{n}\right)$

where $0<a_{n}<1, n=1,2, \ldots$, meaning both the input and output of Logistic mapping is distributed in $(0,1)$. Compared with Tent mapping, it is a nonlinear mapping, and the segmented nonlinear Logistic mapping is defined as follows [20],

$$
a_{n+1}=\left\{\begin{array}{lc}
4 \mu \cdot a_{n} \cdot\left(0.5-a_{n}\right), & 0 \leq a_{n}<0.5 \\
1-4 \mu \cdot\left(a_{n}-0.5\right) \cdot\left(1-a_{n}\right), & 0.5 \leq a_{n}<1
\end{array}\right.
$$

In globally convergent PSO algorithm, if the initial population is inappropriate, the result will possibly reach the local optimum. To solve the convergence problem affected by initial population, this paper adopts double mapping strategy to generate the initial population for the PSO algorithm due to the randomness and sensitiveness to the initial value of chaotic mapping.

This paper takes the chaotic mapping mentioned above to generate the velocity and position of initial population, includes three steps following.

1) Firstly, the random sector $1 \cdot D$ of position $X_{1}$ and velocity $V_{1}$ of one of two particles representing the population is generated and each subsection of the vector is valued among $(0,1)$.

2) $2 \cdot(N-1)$ vectors are generated by iteration of equation (15) and (16), representing the position $X_{2}, X_{3}, \ldots, X_{N}$ and velocity $V_{2}, V_{3}, \ldots, V_{N}$ of the $N-1$ particles left.

$X(i+1, d)=\left\{\begin{array}{c}4 \cdot 4 X(i-1, d)(0.5-X(i-1, d)) \\ 0<X(i-1, d)<0.5 \\ 1-4 \cdot 4(1-X(i-1, d))(X(i-1, d)-0.5), \\ 0.5 \leq X(i-1, d)<1\end{array}\right.$

$V(i+1, d)=\left\{\begin{array}{c}4 \cdot 4 V(i-1, d)(0.5-V(i-1, d)), \\ 0<V(i-1, d)<0.5 \\ 1-4 \cdot 4(1-V(i-1, d))(V(i-1, d)-0.5), \\ 0.5 \leq V(i-1, d)<1\end{array}\right.$

3) Because the initial value is among $(0,1)$ from these two steps, different optimization problems require different optimization interval. Therefore, we map the 
initial position and velocity from above steps into the appointed search space $\left(-S_{i d}, S_{i d}\right)$ according to (17).

$$
\left\{\begin{array}{l}
X(i, d)=S_{i d} \cdot(2 \cdot X(i, d)-1) \\
V(i, d)=S_{i d} \cdot(2 \cdot V(i, d)-1)
\end{array}\right.
$$

It is completed with double mapping in three steps to generate initial population of PSO algorithm.

\subsection{Particle Fitness Optimization Based on Evolutional Status Prediction}

To avoid the premature convergence due to the inappropriate fitness value, this paper takes evolutionary state identification strategy to select the fitness value, including following steps.

1) In each stage of population evolution, the mean Euclidean distance between each particle and other particles is calculated as its mean distance under current evolutionary state.

$d_{i}=\frac{1}{N-1} \sum_{j=1, j \neq i}^{N} \sqrt{\sum_{k=1}^{D}\left(x_{i}^{k}-x_{j}^{k}\right)^{2}}$

2) The mean distance between the global optimal particle and other particles is labelled as $d_{g}$, and the maximum distance $d_{\max }$ and minimum mean distance $d_{\min }$ is selected to calculate the evolutionary factor fof current state.

$f=\frac{d_{g}-d_{\text {min }}}{d_{\text {max }}-d_{\text {min }}} \in[0,1]$

3) Different evolutionary factors represent different evolutionary states, divided into four state sets: detection, development, convergence, and exit denoted as $S_{1}, S_{2}, S_{3}$ and $S_{4}$, respectively. Because the division of evolutionary factor is fuzzy and uncertain, this paper chooses fuzzy classification method to classify the evolutionary state of population.

I. $S_{1}$ :At the beginning of algorithm, the particles in initial population are dispersed without the spatial feature of the solution, therefore, the membership function is defined as,

$\mu S_{1}(f)=\left\{\begin{array}{lr}0, & 0 \leq f \leq 0.4 \\ 5 \cdot f-2, & 0.4<f \leq 0.6 \\ 1, & 0.6<f \leq 0.7 \\ -10 \cdot f+8, & 0.7<f \leq 0.8 \\ 0, & 0.8<f \leq 1\end{array}\right.$

II. $S_{2}$ :After ceaseless iteration, the solution space region is approaching, the particles in the population will be close to the optimal region. At this time, the membership function is defined as,

$$
\mu S_{2}(f)=\left\{\begin{array}{lr}
0, & 0 \leq f \leq 0.2 \\
10 \cdot f-2, & 0.2<f \leq 0.3 \\
1, & 0.3<f \leq 0.4 \\
-5 \cdot f+3, & 0.4<f \leq 0.6 \\
0, & 0.6<f \leq 1
\end{array}\right.
$$

III. $S_{3}$ :All the particles will collect together around optimal solution after iterations, here, the membership function is defined as,

$$
\mu S_{3}(f)=\left\{\begin{array}{lc}
1, & 0 \leq f \leq 0.1 \\
-5 \cdot f+1.5, & 0.1<f \leq 0.3 \\
0, & 0.3<f \leq 1
\end{array}\right.
$$

IV. $S_{4}$ : When the algorithm is constrained to the local optimum, the real global optimal solution will deviate the centre of current population, then the membership function is defined as,

$\mu S_{4}(f)=\left\{\begin{array}{lc}1, & 0 \leq f \leq 0.7 \\ 5 \cdot f-3.5, & 0.7<f \leq 0.9 \\ 1, & 0.9<f \leq 1\end{array}\right.$

From Eqs. (20) \& (23), we can see that when the population stays at the transition stage, two membership functions are activated. It has two evolutionary states if only judged by the $f$, thus we cannot determine the final state.

\subsection{Inertia Weight Optimization Based on Nonlinear Decrease}

The inertia factor $\omega$ of PSO algorithm is one of the parameters of great importance. In order to possess better dynamic property and help search the optimal solution in the global and local search area, this paper takes the nonlinear decrease strategy to optimize it.

$$
\begin{aligned}
\omega_{i}= & \left(\omega_{\text {start }}-\omega_{\text {end }}\right)\left(t_{i} / t_{\max }\right)^{2} \\
& +\left(\omega_{\text {start }}-\omega_{\text {end }}\right)\left(2 t_{i} / t_{\max }\right)+\omega_{\text {start }}
\end{aligned}
$$

where the maximum iteration number is denoted as $t_{\max }$, $t_{i}$ is current iteration time, $\omega_{\text {start }}$ and $\omega_{\text {end }}$ is the maximum and minimum value of inertia factor. Similarly, the inertia factor is firstly valued with a maximum $\omega_{\text {start }}$, and then nonlinearly decreases with the iteration. The $\omega$ value of each stage is calculated by (24) until the minimum $\omega_{\text {end }}$ is reached.

In addition, to reach the local optimal solution as quickly as possible, this paper makes improvement on the global and local search ability in the iteration period. Thus, the velocity equation is adjusted to another one with inertia factor.

$$
\begin{aligned}
v_{i d}(t+1)= & \omega v_{i d}(t)+c_{1} r_{1}\left(p_{i d}(t)-x_{i d}(t)\right) \\
& +c_{2} r_{2}\left(n p_{i d}(t)-x_{i d}(t)\right)
\end{aligned}
$$


Therefore, it can have good performance in searching the optimal with a higher accuracy and faster convergence speed.

\subsection{Particle Iteration Based on Genetic Operator}

After the inertia weight is optimized by nonlinear decrease, the PSO algorithm is further optimized by hybridization of genetic algorithm. Firstly, we select the particles with a certain of determined crossover probability by users. Then, random assortment between particles is conducted to generate children particles of same amount, and replace the parent particles so as to keep constant the particle number. The updating equation for the particle position is,

$\operatorname{child}_{1}(\vec{X})=\vec{P} \cdot \operatorname{parpent}_{1}(\vec{X})+(1-\vec{P}) \cdot \operatorname{parpent}_{2}(\vec{X})$

$\operatorname{child}_{2}(\vec{X})=\vec{P} \cdot \operatorname{parpent}_{2}(\vec{X})+(1-\vec{P}) \cdot \operatorname{parpent}_{1}(\vec{X})$

where $\vec{X}$ is $d$ dimensional position vector, $\operatorname{child}_{k}(\stackrel{1}{X})$ and $\operatorname{parpent}_{k}(\vec{X}), k=1,2$ represents the position of child particle and parent particle, respectively; $\vec{P}$ is $d$-dimensional uniformly distributed random vector, which is valued among $[0,1]$. The velocity of particle is updated from equations below.

$$
\begin{aligned}
& \operatorname{child}_{1}(\vec{V})=\frac{\operatorname{parpent}_{1}(\vec{V})+\operatorname{parpent}_{2}(\vec{V})}{\left|\operatorname{parpent}_{1}(\vec{V})+\operatorname{parpent}_{2}(\vec{V})\right|} \cdot\left|\operatorname{parpent}_{1}(\vec{V})\right| \\
& \operatorname{child}_{2}(\vec{V})=\frac{\text { arpent }_{1}(\vec{V})+\text { parpent }_{2}(\vec{V})}{\mid \text { parpent }_{1}(\vec{V})+\text { parpent }_{2}(\vec{V}) \mid} \cdot\left|\operatorname{parpent}_{2}(\vec{V})\right|
\end{aligned}
$$

where $\vec{V}$ is $d$ dimensional position vector, $\operatorname{child}_{k}(\vec{V})$ and $\operatorname{parpent}_{k}(\vec{V}), \quad k=1,2$ represents the velocity of child particle and parent particle, respectively.

\section{Simulation Experiment}

To verify the effectiveness of the improved algorithm, this paper conducted the simulation experiment. It chose three typical function optimization problems to test the performance of improved PSO algorithm. In this paper, the initial population size is set as Size $=100$, learning factor $c_{1}=c_{2}=1.5, \quad \omega_{\text {start }}=0.9, \quad \omega_{\text {end }}=0.9, \quad$ maximum iteration step $T=100$. Three typical functions are shown as follows.

1) Sphere function,

$$
f_{1}(x)=\sum_{i}^{n} x_{i}^{2}
$$

Global optimum point, $x_{i}=0, f_{1}(x)=0,-100 \leq x_{i} \leq 100$.

2) Rosenbrock function,

$$
f_{2}(x)=\sum_{i=1}^{n}\left(100 \cdot\left(\mathrm{x}_{\mathrm{i}+1}-\mathrm{x}_{\mathrm{i}}^{2}\right)^{2}+\left(\mathrm{x}_{\mathrm{i}}-1\right)^{2}\right), \quad-30 \leq \mathrm{x}_{\mathrm{i}} \leq 30
$$

Its optimal state and optimal value is,

$\min f\left(x^{*}\right)=f(1,1, \mathrm{~K}, 1)=0$

3) Rastrigrin function,

$$
f_{3}(x)=\sum_{i=1}^{n}\left(x_{i}^{2}-10 \cos \left(2 \pi \mathrm{x}_{\mathrm{i}}\right)+10\right), \quad-5.12 \leq \mathrm{x}_{\mathrm{i}} \leq 5.12
$$

Its optimal state and value is,

$$
f_{3}(x)=\sum_{i=0}^{n} f(0,0, \mathrm{~K}, 0)=0
$$

\begin{tabular}{|c|c|c|c|c|}
\hline Functions & Optimization & Average & $\begin{array}{l}\text { Success } \\
\text { rate } / \%\end{array}$ & $\begin{array}{c}\text { Execution } \\
\text { time / s }\end{array}$ \\
\hline \multirow{2}{*}{ Sphere } & PSO & $\begin{array}{c}1.45 \times 10- \\
55\end{array}$ & 80.6 & 10.33 \\
\hline & IM-PSO & $\begin{array}{c}1.06 \times 10- \\
77\end{array}$ & 93.7 & 5.66 \\
\hline \multirow{2}{*}{ Rosenbrock } & PSO & $\begin{array}{c}10.31 \times 10- \\
1\end{array}$ & 86.2 & 11.03 \\
\hline & IM-PSO & $\begin{array}{c}2.46 \times 10- \\
10\end{array}$ & 94.8 & 9.11 \\
\hline \multirow{2}{*}{ Rastrigin } & PSO & $2.95 \times 10-1$ & 76 & 18.6 \\
\hline & IM-PSO & $1.46 \times 10-3$ & 94.9 & 17.1 \\
\hline
\end{tabular}

Simulation experiment is conducted on a computer with 2.8GHZ CPU, $2 \mathrm{G}$ memory and Matlab 7.0. The simulation results are listed in Table 1 and shown in Figs. 1-3.

Tab. 1. The results for the test.

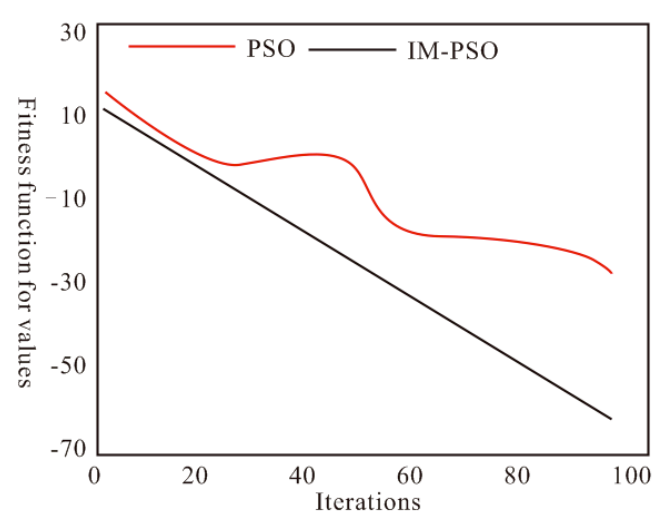

Fig. 1. The Rosenbrock function optimization process.

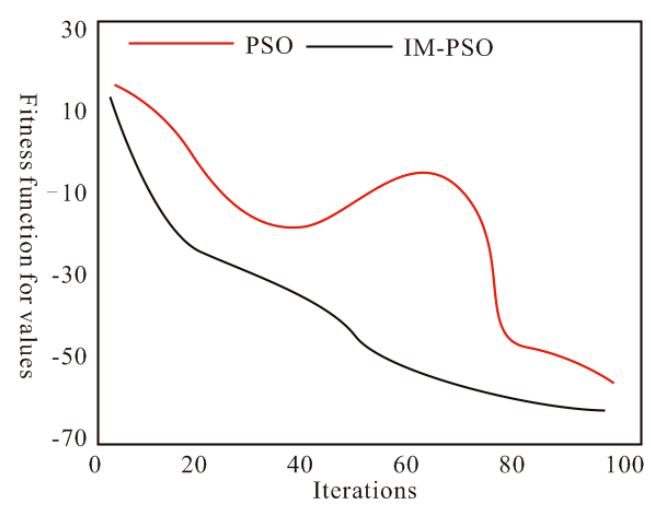

Fig. 2. Rastrigin function optimization process. 
Compared with original PSO algorithm, the improved PSO algorithm presented in this paper is faster, more stable and easier to reach the optimum value.

Then, this improved PSO algorithm is used for parameter estimation firstly under the condition without noise, shown in Fig. 4.

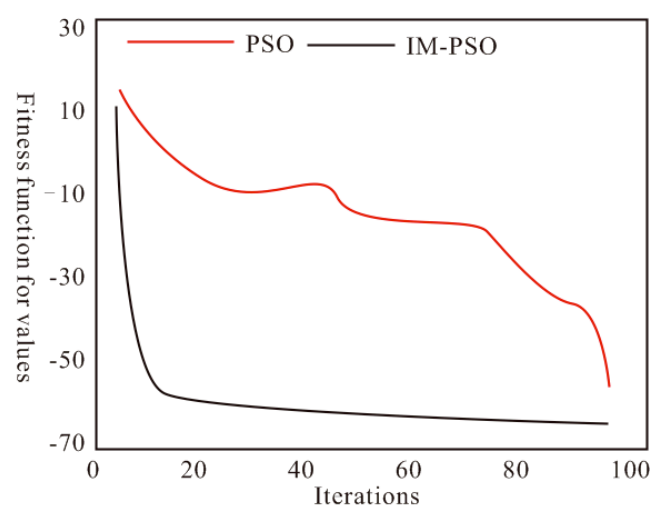

Fig. 3. the Sphere function optimization process.

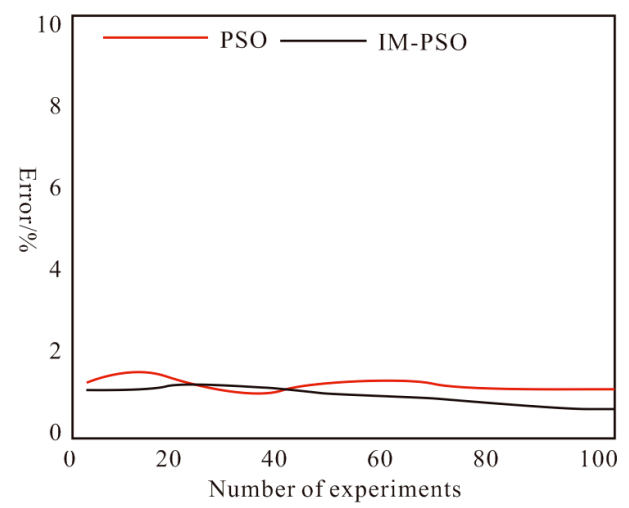

Fig. 4. Parameters noiseless case compared with the estimated.
Taking into consideration of noises, the standard state variable $(x, y, z)$ is superimposed by white noises in $[-0.1,0.1]$ with the comparable results in Figure 5.

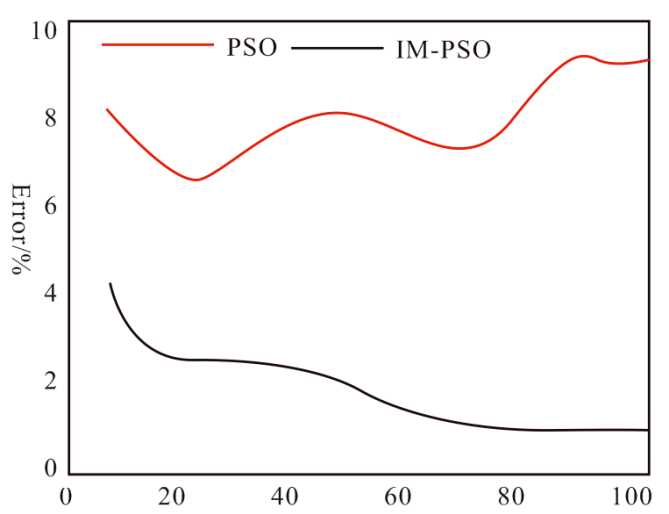

Fig. 5. Parameters are compared with the estimated noise case.

From the simulation result, the parameter estimation model of chaotic control system based on improved PSO algorithm shows little difference with standard PSO algorithm under the condition without noise, but have better robustness under noises.

\section{Conclusion}

There exist a lot of problems in current chaotic control and synchronous control methods, such as incomplete state variables of chaotic system, partially unknown or totally unknown parameters, and disturbance in systematic parameters. This paper proposes a parameter estimation model of chaotic control system based on improve PSO algorithm. Simulation results show that the proposed model has higher accuracy and stronger robustness in parameter estimation.

\section{References}

1. L. Lezhu, A chaotic secure communications method based on chaotic systems partial series parameter estimation, Acta Phys. Sin., pp.24-29 (2014).

2. W. Liu, Evolutionary modelling for parameter estimation for chaotic system, Acta Phys. Sin., pp.447-456 (2014).

3. H. Jianhong, Synchronization of uncertain chaotic systems and parameters identification, Journal of Hebei Normal Univeristy, pp. 30-35 (2014).

4. H. Longlong, Chaos control of permanent magnet synchronous motor with parameter uncertainties based on adaptive sliding mode, Modular Machine Tool \& Automatic Manufacturing Technique, pp.55-57 (2013).

5. D. Honggang, Synchronization and chaos control of a chaotic complex system, Journal of Sichuan University (Natural Science Edition), pp. 1049-1052 (2013).

6. C. Zhongyong, Parameter estimation and noise filter in chaotic measurement, Acta Metrologica Sinica, pp.497-501 (2013).

7. X. Chenggang, Computation of chaotic oscillation parameter in ship power system based on RHR algorithm, Ship Science and Technology, pp.86-90 (2014).

8. L. Kunhua, Adaptive synchronization of fractional order LV chaotic system with uncertain parameters, Journal of Lanzhou University of Technology, pp.164-167 (2013).
9. D. Jun, Function projective synchronization and parameter identification of different fractional-order Hyper-chaotic systems, Journal of Electronics \& Information Technology, pp.1371-1375 (2013).

10. G. Zhihua, Generalized function lag projective synchronization and parameter identification of a class of chaotic systems with fully uncertain parameters, Journal of Henan University (Natural Science), pp. 311-314 (2013).

11. W. Hengdong, Parameters estimation of chaotic telemetry system between two different time-delayed chaotic systems, Telecommunication Engineering, pp. 565-569 (2013).

12. C. Zongping, Chaotic system parameter identification based on chaotic fish-swarm algorithm, Science Technology and Engineering, pp. 23-29 (2013)

13. D. Honggang, Chaos synchronization and parameter identification of a new fractional system, Fuzzy Systems and Mathematics, pp.23-29 (2013).

14. L. Heng, Parameter identification and backstepping design of adaptive fuzzy controller for Feng chaotic system, Chinese Journal of Scientific Instrument, pp. 2464-2470 (2013).

15. Z. Hongli, Chaotic system parameter identification based on quantum particle swarm optimization algorithm, Acta Phys. Sin., pp.106-111 (2013). 
16. C. Jun, Structural modal parameter identification based on quantum-behaved particle swarm optimization, Journal of Vibration and Shock, pp.72-76 (2014).

17. J. Ping, Task scheduling algorithm and application based on hybrid discrete particle swarm optimization, Fire Control \& Command Control, pp.146-149 (2014).

18. H. Shuangshuang, A pseudo-random number generator using three dimensional chaotic map, Journal of Huazhong University of Science And Technology, pp.16-19 (2013).
19. L. Jinglin, A new approach to analyze discrete chaotic maps stability based on k-error exhaustive entropy, Telecommunication Engineering, pp.170-174 (2014).

20. P. Jun, A block encryption algorithm combined with the discrete chaotic map and Feistel network, Journal of Electronics \& Information Technology, pp.707-711 (2013). 